\title{
Non-zero density QCD by the Taylor expansion method: The isentropic equation of state, hadronic fluctuations and more
}

\author{
Chuan Miao and Christian Schmidt* (RBC-Bielefeld Collaboration) \\ Department of Physics, Brookhaven National Laboratories, Upton, NY 11733, USA. \\ Fakultät für Physik, Universität Bielefeld, D-33615 Bielefeld, Germany. \\ E-mail: chuan@quark.phy.bnl.gov, schmidt@physik.uni-bielefeld.de
}

\begin{abstract}
We discuss the Taylor expansion approach to non-zero baryon chemical potential $\left(\mu_{B}\right)$ and present results on expanion coefficients of the pressure and energy density up to the 6-th order in $\mu_{B}$. Calculations have been performed with (2+1)-flavor of improved staggerd fermions (p4fat3) and almost physical masses on lattices with temporal extent of four and six time slices. We use the expansion coefficients to construct the isentropic equation of state on lines of constant entropy per baryon number and various different hadronic fluctuations. Furthermore, we estimate the radius of convegence of our expansion, which can be seen as a method to analyze the structure of the QCD phase diagram.
\end{abstract}

The XXVI International Symposium on Lattice Field Theory

July 14-19 2008

Williamsburg, Virginia, USA

\footnotetext{
* Speaker.
} 

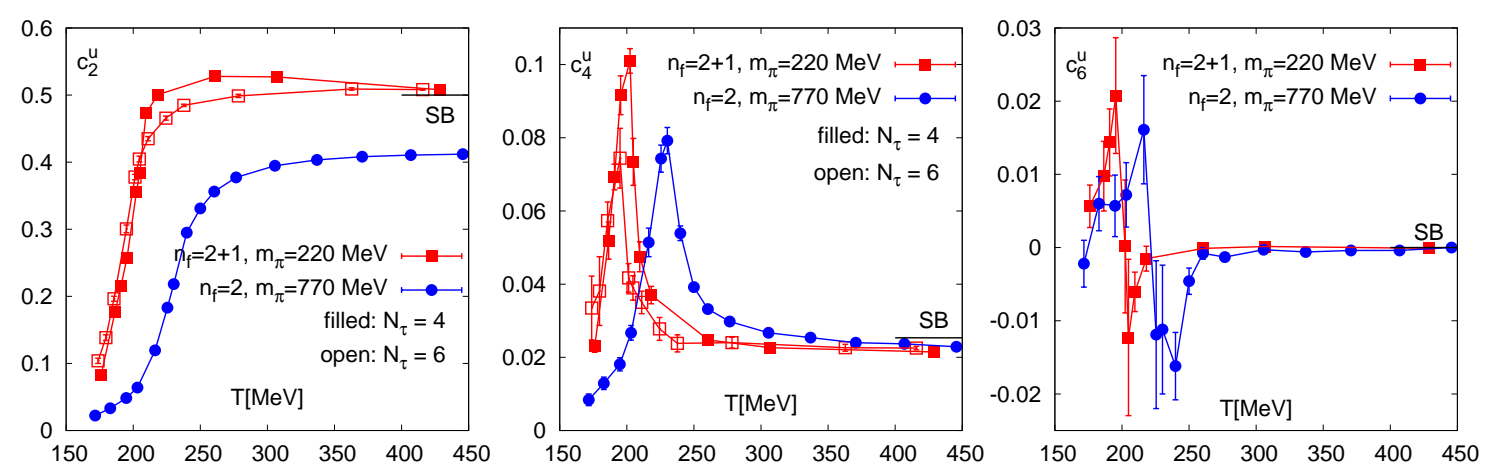

Figure 1: Taylor coefficients of the pressure in term of the up-quark chemical potential. Results are obtained with the p4fat 3 action on $N_{\tau}=4$ (full) and $N_{\tau}=6$ (open symbols) lattices. We compare preliminary results of (2+1)-flavor a pion mass of $m_{\pi} \approx 220 \mathrm{MeV}$ to previous results of 2-flavor simulations with a corresponding pion mass of $m_{p} \approx 770$ [2].

\section{Introduction}

A detailed and comprehensive understanding of the thermodynamics of quarks and gluons, e.g. of the equation of state is most desirable and of particular importance for the phenomenology of relativistic heavy ion collisions. Lattice regularized QCD simulations at non-zero temperatures have been shown to be a very successful tool in analyzing the non-perturbative features of the quark-gluon plasma. Driven by both, the exponential growth of the computational power of recent super-computer as well as by drastic algorithmic improvements one is now able to simulate dynamical quarks and gluons on fine lattices with almost physical masses.

At non-zero chemical potential, lattice QCD is harmed by the "sign-problem", which makes direct lattice calculations with standard Monte Carlo techniques at non-zero density practically impossible. However, for small values of the chemical potential, some methods have been successfully used to extract information on the dependence of thermodynamic quantities on the chemical potential. For a recent overview see, e.g. [1].

\section{The Taylor expansion method}

We closely follow here the approach and notation used in Ref. [绍]. We start with a Taylor expansion for the pressure in terms of the quark chemical potentials

$$
\frac{p}{T^{4}}=\sum_{i, j, k} c_{i, j, k}^{u, d, s}(T)\left(\frac{\mu_{u}}{T}\right)^{i}\left(\frac{\mu_{d}}{T}\right)^{j}\left(\frac{\mu_{s}}{T}\right)^{k}
$$

The expansion coefficients $c_{i, j, k}^{u, d, s}(T)$ are computed on the lattice at zero chemical potential, using stochastic estimators. Some details on the computation are given in [3, 4]. Details on our current data set and the number of random vectors used for the stochastic random noise method are summarized in Table 1 .

In Fig. 1 we show results on the diagonal expansion coefficients with respect to the up-quark 


\begin{tabular}{|c|c|c|c||c|c|c|c|}
\hline \multicolumn{5}{|c||}{$N_{\tau}=4$} & \multicolumn{4}{c|}{$N_{\tau}=6$} \\
\hline T[MeV] & \#Conf. & Sep. & \#r.v. & T[MeV] & \#Conf. & Sep. & \#r.v. \\
\hline \hline 176.04 & 1013 & 20 & 480 & 173.82 & 985 & 10 & 400 \\
186.41 & 1550 & 30 & 480 & 179.63 & 910 & 10 & 400 \\
190.83 & 1550 & 30 & 480 & 185.64 & 1043 & 10 & 400 \\
195.37 & 1550 & 30 & 384 & 194.97 & 924 & 10 & 400 \\
202.42 & 1350 & 30 & 384 & 201.35 & 873 & 10 & 350 \\
204.83 & 475 & 60 & 384 & 204.58 & 717 & 10 & 200 \\
209.63 & 264 & 60 & 384 & 211.11 & 690 & 10 & 150 \\
218.18 & 365 & 30 & 384 & 224.34 & 560 & 10 & 150 \\
260.74 & 199 & 60 & 192 & 237.72 & 670 & 10 & 100 \\
306.88 & 302 & 60 & 96 & 278.36 & 540 & 10 & 50 \\
428.66 & 618 & 10 & 48 & 362.87 & 350 & 10 & 50 \\
& & & & 415.83 & 345 & 10 & 50 \\
\hline
\end{tabular}

Table 1: Details on the calculation: The columns give from left to right the temperature values, the number of evaluated configurations, the number of trajectories by which these configurations are separated and the number of random vectors used for the evaluation of the traces, for $N_{\tau}=4$ and 6 , respectively.

chemical potential up to the six order $\left(c_{n, 0,0}^{u, d, s}\right.$ with $\left.n=2,4,6\right)$. They have been evaluated on configurations generated for a calculation of the equation of state [5], obtained with (2+1)-flavor of the p4fat 3 action [6] and a pion mass of $m_{\pi} \approx 220 \mathrm{MeV}$. Here the full symbols are from $N_{\tau}=4$ lattices, while the open symbols denote results from $N_{\tau}=6$ lattices. The spatial lattice size has been chosen to be $N_{\sigma}=4 N_{\tau}$, which is known to be large enough in order to suppress finite volume effects. Considering cut-off effects, we find that they are small and of similar magnitude as those found for the trace anomaly [5]. This was already anticipated by the analysis of the cut-off corrections that arises in the free gas limit [7]. Similar results for the expansion coefficients have been also obtained with the asqtad [8] and the standard action [9].

We also compare our preliminary results for (2+1)-flavor QCD and a pion mass of $m_{\pi} \approx$ $220 \mathrm{MeV}$, with previously obtained result of 2-flavor QCD and $m_{\pi} \approx 770 \mathrm{MeV}$ [2] (also p4fat3 and $\left.N_{\tau}=4\right)$. It is apparent from Fig. 1 that the critical temperature for these two particular sets of lattice parameter differ substantially. In fact, the transition temperature decreases from about $225 \mathrm{MeV}$ for the heavier mass calculations to about $200 \mathrm{MeV}$ for the lighter mass calculations. Note, that those $T_{c}$ values are the $N_{\tau}=4$ values, which of course are still influenced by the finite lattice spacing. Furthermore, we find from Fig. 11 that the quark number fluctuations of second, fourth and sixth order, which are related to these expansion coefficients, increase with decreasing quark mass.

Alternatively to the quark chemical potentials one can introduce chemical potentials for the conserved quantities baryon number $B$, electric charge $Q$ and strangeness $S\left(\mu_{B, Q, S}\right)$, which are related to $\mu_{u, d, s}$ via

$$
\mu_{u}=\frac{1}{3} \mu_{B}+\frac{2}{3} \mu_{Q}, \quad \mu_{d}=\frac{1}{3} \mu_{B}-\frac{1}{3} \mu_{Q}, \quad \mu_{s}=\frac{1}{3} \mu_{B}-\frac{1}{3} \mu_{Q}-\mu_{S}
$$



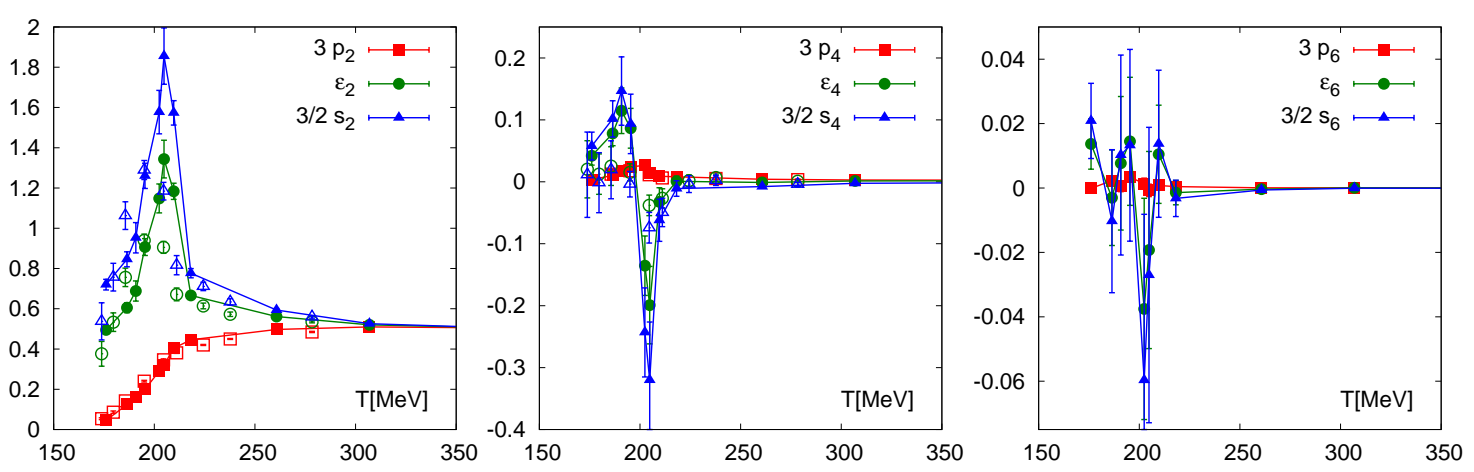

Figure 2: Taylor coefficients of the pressure, the energy and entropy density with respect to the baryon chemical potential. Results are obtained with the p4fat 3 action on $N_{\tau}=4$ (full) and $N_{\tau}=6$ (open symbols) lattices.

By means of these relations the coefficients $c_{i, j, k}^{B, Q, S}$ of the pressure expansion in terms of $\mu_{B, Q, S}$ are easily obtained, in analogy to Eq. 2.1.

$$
\frac{p}{T^{4}}=\sum_{i, j, k} c_{i, j, k}^{B, Q, S}(T)\left(\frac{\mu_{B}}{T}\right)^{i}\left(\frac{\mu_{Q}}{T}\right)^{j}\left(\frac{\mu_{S}}{T}\right)^{k} .
$$

For the rest of this article we will restrict ourselves to the case of $\mu_{Q} \equiv \mu_{S} \equiv 0$, thus we will suppress in the following the indices that are related to these chemical potentials. From the pressure we immediately obtain the baryon number density $n_{B}$, which is given by the derivative of $p / T^{4}$ with respect to the baryon chemical potential $\mu_{B}$ and can be expressed in term of the expansion coefficients $c_{n}^{B}$, we have

$$
\frac{n_{B}}{T^{3}}=\sum_{n=2}^{\infty} n c_{n}^{B}(T)\left(\frac{\mu_{B}}{T}\right)^{n-1}
$$

Using standard thermodynamic relations we can also calculate the expansion coefficients of the trace anomaly or equivalently the difference between energy density and three times the pressure,

$$
\frac{\varepsilon-3 p}{T^{4}}=\sum_{n=0}^{\infty} \bar{c}_{n}^{B}(T)\left(\frac{\mu_{B}}{T}\right)^{B}
$$

where the expansion coefficients $\bar{c}_{n}^{B}$ are given by

$$
\bar{c}_{n}^{B}(T)=T \frac{\mathrm{d} c_{n}^{B}(T)}{\mathrm{d} T}
$$

Combining Eqs. 2.3, 2.5, and 2.6 we then obtain the Taylor expansions for the energy and entropy densities [10]

$$
\begin{aligned}
\frac{\varepsilon}{T^{4}} & =\sum_{n=0}^{\infty}\left(3 c_{n}^{B}(T)+\bar{c}_{n}^{B}(T)\right)\left(\frac{\mu_{B}}{T}\right)^{n} \equiv \sum_{n=0}^{\infty} \varepsilon_{n}\left(\frac{\mu_{B}}{T}\right)^{n} \\
\frac{s}{T^{3}} & \equiv \frac{\varepsilon+p-\mu_{B} n_{B}}{T^{4}}=\sum_{n=0}^{\infty}\left((4-n) c_{n}^{B}(T)+\bar{c}_{n}^{B}(T)\right)\left(\frac{\mu_{B}}{T}\right)^{n} \equiv \sum_{n=0}^{\infty} s_{n}\left(\frac{\mu_{B}}{T}\right)^{n} .
\end{aligned}
$$


At present, we calculate the expansion coefficients $\bar{c}_{n}^{B}$ from the coefficients $c_{n}^{B}$, in accordance with Eq. 2.6, by performing the $T$ derivative numerically, which introduces a small systematic error.

In Fig. 2 we show the second, fourth and sixth order expansion coefficients of the pressure, energy density and entropy density as given in Eqs. 2.3 and 2.8, obtained with the p4fat 3 action. Full symbols are from $N_{\tau}=4$ lattices, while the open symbols denote results from $N_{\tau}=6$ lattices. We again find small cut-off effects, however, higher order derivatives of pressure, energy density and entropy density with respect to $\mu_{B}$ are still very preliminary, as the error bars are large. This is especially true for the results from $N_{\tau}=6$ lattices. Nevertheless, the overall pattern of the coefficients is in agreement with expectations based on an analysis of the singular behavior of the free energy, making use of an appropriate scaling Ansatz.

We find that the magnitude of the coefficients is decreasing drastically with increasing order, for all analyzed temperatures. Thus an approximation of the equation of state for small baryon chemical potential by means of a fourth or sixth order expansion seems to be justified. In general, an analysis of the radius of convergence of such a Taylor series is of great interest for an analysis of the QCD phase diagram, since the radius of convergence is bounded by the location of the QCD critical point as well as by any first order phase transition line (see Sec. 5).

\section{The isentropic equation of state}

By using the Taylor expansion coefficients of the baryon number (Eq. 2.4) and entropy density (Eq. 2.8), we can compute the ratio of entropy per baryon number as function of $T$ and $\mu_{B}$. The 0 -th order coefficient for the entropy density has been taken from [5]. Solving numerically for a constant ratio of entropy per baryon number, $s / n_{B}$, we determine isentropic trajectories in the $\left(T, \mu_{B}\right)$-plane. These trajectories are relevant for the description of matter created in relativistic heavy ion collisions. After equilibration the dense medium created in such a collision will expand along lines of constant entropy per baryon. It then is of interest to calculate thermodynamic quantities along such isentropic lines.

We find that isentropic expansion at high temperature is well represented by lines of constant $\mu_{B} / T$ down to temperatures close to the transition, $T \simeq 1.2 T_{0}$. In the low temperature regime we observe a bending of the isentropic lines in accordance with the expected asymptotic low temperature behavior. The isentropic expansion lines for matter created at SPS correspond to $s / n_{B} \simeq 45$ while the isentropes at RHIC correspond to $s / n_{B} \simeq 300$. The energy range of the AGS which also corresponds to an energy range relevant for future experiments at FAIR/Darmstadt is well described by $s / n_{B} \simeq 30$. These lines are shown in Fig. 3 (left) together with data points characterizing the chemical freeze-out of hadrons measured at AGS, SPS and RHIC energies. These data points have been obtained by comparing experimental results for yields of various hadron species with hadron abundances in a resonance gas [11, 12]. The solid curve shows a phenomenological parameterization of these freeze-out data [12]. In general our findings for lines of constant $s / n_{B}$ are in good agreement with phenomenological model calculations that are based on combinations of ideal gas and resonance gas equations of state at high and low temperature, respectively [13, 14].

Results shown in Fig. 3 are based on a fourth order expansion of the pressure. We find, however, that the truncation error is small, i.e. the results change only little when we consider also the sixth order term in $\mu_{B}$. In accordance with the good convergence of our results, we find, that all 

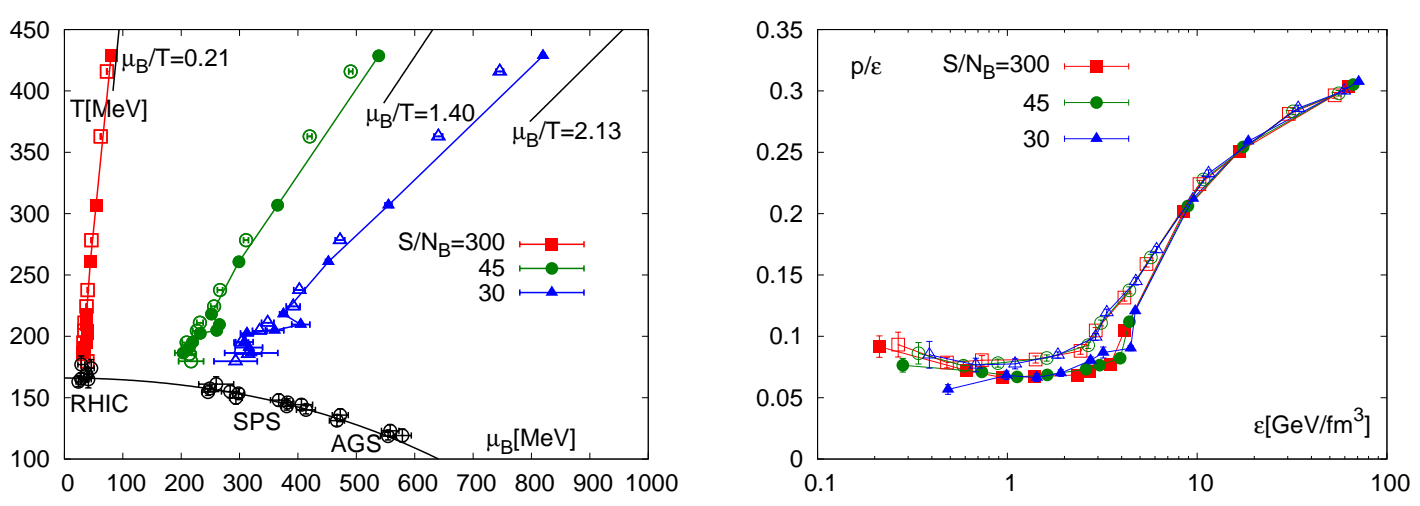

Figure 3: On the left panel we show Isentropic trajectories in the $\left(T-\mu_{B}\right)$-diagram, corresponding to $s / n_{B}=300,45,30$ respectively. Open symbols are form $N_{\tau}=6$, obtained by a 4th order Taylor expansion of the pressure. Filled symbols are from $N_{\tau}=4$ calculations. We also show freeze-out data, as well as a parameterization of the freeze-out curve from [12]. Corresponding free gas limits are $\mu_{B} / T=0.21,1.40,2.13$ respectively and are indicated by solid lines. On the right panel we plot the the ratio of pressure and energy density along those trajectories.

trajectories shown in Fig. B (left) are well within the radius of convergence of the Taylor series. At present we estimate the radius of convergence of the pressure series to $\left(\mu_{B} / T\right)^{\text {crit }} \gtrsim 2.7$. The cut-off effects can be estimated by comparing open and full symbols.

We now proceed and calculate energy density and pressure on lines of constant entropy per baryon number using our Taylor expansion results up to $\mathscr{O}\left(\mu_{B}^{4}\right)$. Again we take the 0 -th order coefficients from [5]. We find that both quantities obtain corrections of about $10 \%$ at AGS (FAIR) energies $\left(s / n_{B}=30\right)$ and high temperatures. The dependence of $\varepsilon$ and $p$ on $s / n_{B}$ cancels to a large extent in the ratio $p / \varepsilon$, which is most relevant for the analysis of the hydrodynamic expansion of dense matter. This may be seen by considering the leading $\mathscr{O}\left(\mu_{B}^{2}\right)$ correction,

$$
\frac{p}{\varepsilon}=\frac{1}{3}-\frac{1}{3} \frac{\varepsilon_{0}-3 p_{0}}{\varepsilon_{0}}\left(1+\left[\frac{\bar{c}_{2}}{\varepsilon_{0}-3 p_{0}}-\frac{\varepsilon_{2}}{\varepsilon_{0}}\right]\left(\frac{\mu_{B}}{T}\right)^{2}\right) .
$$

In Fig. 3 (right) we show $p / \varepsilon$ as function energy density along our three isentropic trajectories. The softest point of the equation of state is found to be $(p / \varepsilon)_{\min } \simeq 0.07-0.09$, for $N_{\tau}=4$ and 6 respectively. Within our current numerical accuracy it is independent of $s / n_{B}$. Similar results for the asqtad action have been obtained in [8]. However, as our data is preliminary, the analysis clearly suffers from insufficient statistics, which is in particular true for our $N_{\tau}=6$ results.

\section{Hadronic Fluctuations}

Quark number fluctuations are related to the derivatives of QCD partition function with respect to the quark chemical potentials by the fluctuation-dissipation theorem. The Taylor expansion coefficients $c_{i, j, k}^{u, d, s}$, as defined in Eq. 2.1, can thus be directly interpreted as quark number fluctuations at $\mu=0$. However, quark fluctuations can not be detected directly in experiments due to confinement. Therefore we will consider fluctuations in terms of hadronic quantum numbers, i.e. baryon 

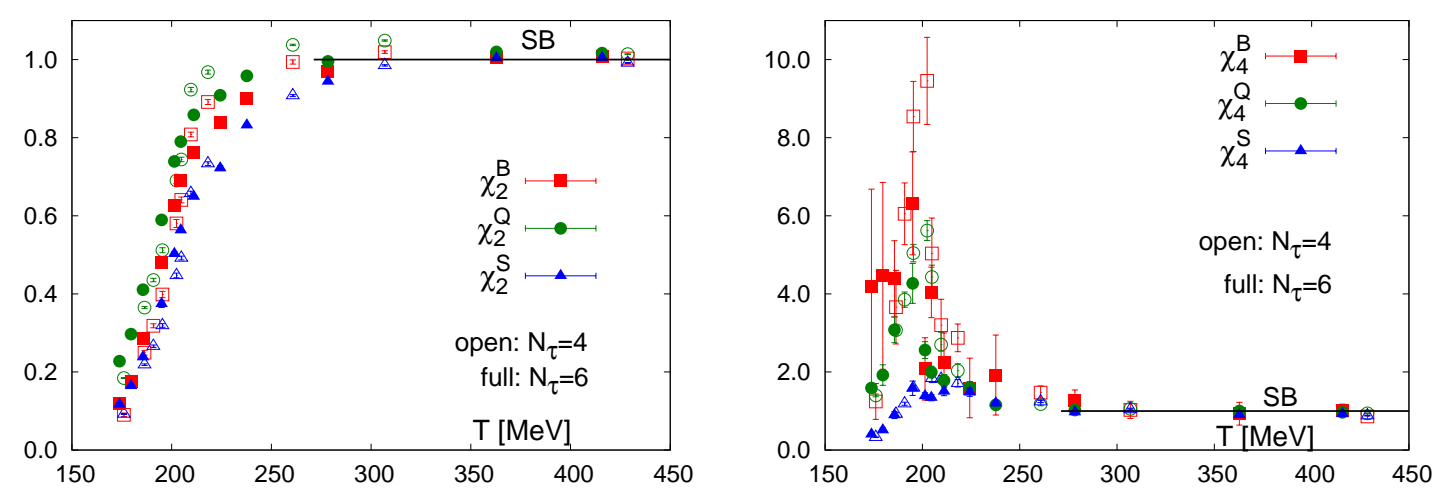

Figure 4: Quadratic and quartic fluctuations of baryon number, electric charge and strangeness, normalized by their corresponding Stefan-Boltzmann value. The results on $N_{\tau}=4$ lattices (open symbols) and $N_{\tau}=6$ lattices (full symbols) are in good agreements.

number $B$, electric charge $Q$ and strangeness $S$, which are more easily obtained by experiment. These fluctuations are related with the hardonic Taylor expansion coefficients $c_{i, j, k}^{B, S}$, as given in Eq. 2.3.

In general, the quadratic fluctuations $\chi_{2}^{X}$ at zero chemical potentials can be obtained from the second order coefficient $c_{2}^{X}$

$$
\left.c_{2}^{X} \equiv \frac{1}{2 V T^{3}} \frac{\partial^{2} \ln Z}{\partial\left(\mu_{X} / T\right)^{2}}\right|_{\mu_{B, Q, S}=0}=\frac{1}{2 V T^{3}}\left\langle\left(\delta N_{X}\right)^{2}\right\rangle_{0},
$$

where $\delta N \equiv N-\langle N\rangle$ denotes the normalized net-density and $\langle\ldots\rangle_{0}$ indicates that the expectation value has been taken at $\mu_{B, Q, S}=0$. Under such conditions, baryon number, electric charge and strangeness vanish, and we have $\delta N=N$. We define

$$
\chi_{2}^{X}=\frac{1}{V T^{3}}\left\langle N_{X}^{2}\right\rangle_{0}=2 c_{2}^{X}
$$

Similarly, we obtain the quartic charge fluctuations by

$$
\chi_{4}^{X}=\frac{1}{V T^{3}}\left(\left\langle N_{X}^{4}\right\rangle_{0}-3\left\langle N_{X}^{2}\right\rangle_{0}^{2}\right)=24 c_{4}^{X},
$$

and correlations among two conserved charges by

$$
\begin{aligned}
& \chi_{11}^{X Y}=\frac{1}{V T^{3}}\left(\left\langle N_{X} N_{Y}\right\rangle_{0}-\left\langle N_{X}\right\rangle_{0}\left\langle N_{Y}\right\rangle_{0}\right)=c_{11}^{X Y}, \\
& \chi_{22}^{X Y}=\frac{1}{V T^{3}}\left(\left\langle N_{X}^{2} N_{Y}^{2}\right\rangle_{0}-\left\langle N_{X}^{2}\right\rangle_{0}\left\langle N_{Y}^{2}\right\rangle_{0}-2\left\langle N_{X} N_{Y}\right\rangle_{0}^{2}\right)=4 c_{22}^{X Y},
\end{aligned}
$$

where $X, Y \in\{B, Q, S\}$.

In Fig. $\bigoplus$ we show results for quadratic and quartic fluctuations of $B, Q$ and $S$. The quadratic fluctuations $\chi_{2}^{B, Q, S}$ rise rapidly in the transition region where the quartic fluctuations $\chi_{4}^{B, Q, S}$ show a peak. The peak height is more pronounced for the baryon number fluctuations than for fluctuations of the strange quarks. 
We compare the results obtained on lattices with temporal extent $N_{\tau}=4$ and 6 . We notice that they are in general compatible with each other, especially in the high temperature phase, where both quadratic and quartic fluctuations approach the Stephan-Boltzmann limit quickly. The transition temperature has been previously determined to be $T_{c}=202 \mathrm{MeV}$ and $196 \mathrm{MeV}$ on $N_{t}=4$ and 6 lattices respectively [15]. We thus conclude that at temperatures of about $1.5 T_{c}$ and higher, quadratic and quartic fluctuations of $B, Q$ and $S$ are well described by the ideal massless quark gas.

At low temperature, hadrons are the relevant degrees of freedom. The hadron resonance gas (HRG) model has been shown to provide a good description of thermal conditions at freeze-out. We thus compare the fluctuations in the low temperature phase with a HRG model, where we include all mesons and baryons with masses smaller than $2.5 \mathrm{GeV}$ from the particle data book.

In Fig. 5, we show the ratio of quartic and quadratic fluctuations for $B, S$ and $Q$. In the HRG model, $\chi_{4}^{B} / \chi_{2}^{B}$ is easily obtained in the Boltzmann approximation, which is valid for a dilute baryonic gas in the temperature range of interest. One finds that all details on the hadron mass spectrum and temperature dependence cancel and the result is a constant, given by the unit square of the baryonic charge (one for all baryons). This is in fact reproduced by the lattice results shown in Fig. 5 (top left).

The ratio of quartic and quadratic fluctuations for $S$ and $Q$ are more complicated even in the Boltzmann limit, since hadrons with different electric/strange charge give rise to different contributions to the corresponding fluctuations. For strangeness fluctuations, shown in Fig. 5 (bottom), the Boltzmann limit is still a good approximation; but for electric charge fluctuations, the pion mass plays an important role. In order to check for the sensitivity of electric charge fluctuations on the pion mass, we show in Fig. 5 (top right) results of a HRG model calculation with physical pion masses and without the pion sector, i.e. for infinitely heavy pions.

In Fig. 6, we show the various correlations $\chi_{11}^{B Q}, \chi_{11}^{B S}$ and $\chi_{11}^{Q S}$ normalized to quadratic fluctuations $\chi_{2}^{B}$ and $\chi_{2}^{Q}$ respectively. The results from $N_{\tau}=4$ and 6 lattices agree with each other very well, and they are compared with the HRG model in the low temperature phase and StephanBoltzmann limit in the high temperature phase. We find that the correlations reproduce the qualitative behaviour of the HRG model below $T_{c}$ and again start to agree with the free gas predictions for $T \gtrsim 1.5 T_{c}$.

\section{Radius of convergence}

Whenever an observable at $\mu_{B}>0$ is approximated by a truncated Taylor series, expanded around $\mu_{B}=0$, an analysis of the convergence behavior of the series is, of course, mandatory. In addition, the convergence radius of such a series, as e.g. given in Eq. 2.3 for the pressure, is of great interest for the structure of the QCD phase diagram. By definition, the radius of convergence is the distance to the closest singularity in the $\left(T, \mu_{B}\right)$-plane. It is bounded by the QCD critical point as well as by any line of first order transitions and could thus provide an interesting method to determine the QCD critical point. This method was proposed in Ref. [3] and was applied in the case of standard staggered fermions in Ref. [9].

One way to evaluate the radius of convergence $\rho$ is by means of the formula

$$
\rho(T)=\lim _{n \rightarrow \infty} \rho_{n}(T), \quad \text { with } \quad \rho_{n}(T)=\sqrt{c_{n}^{B}(T) / c_{n+2}^{B}(T)} .
$$



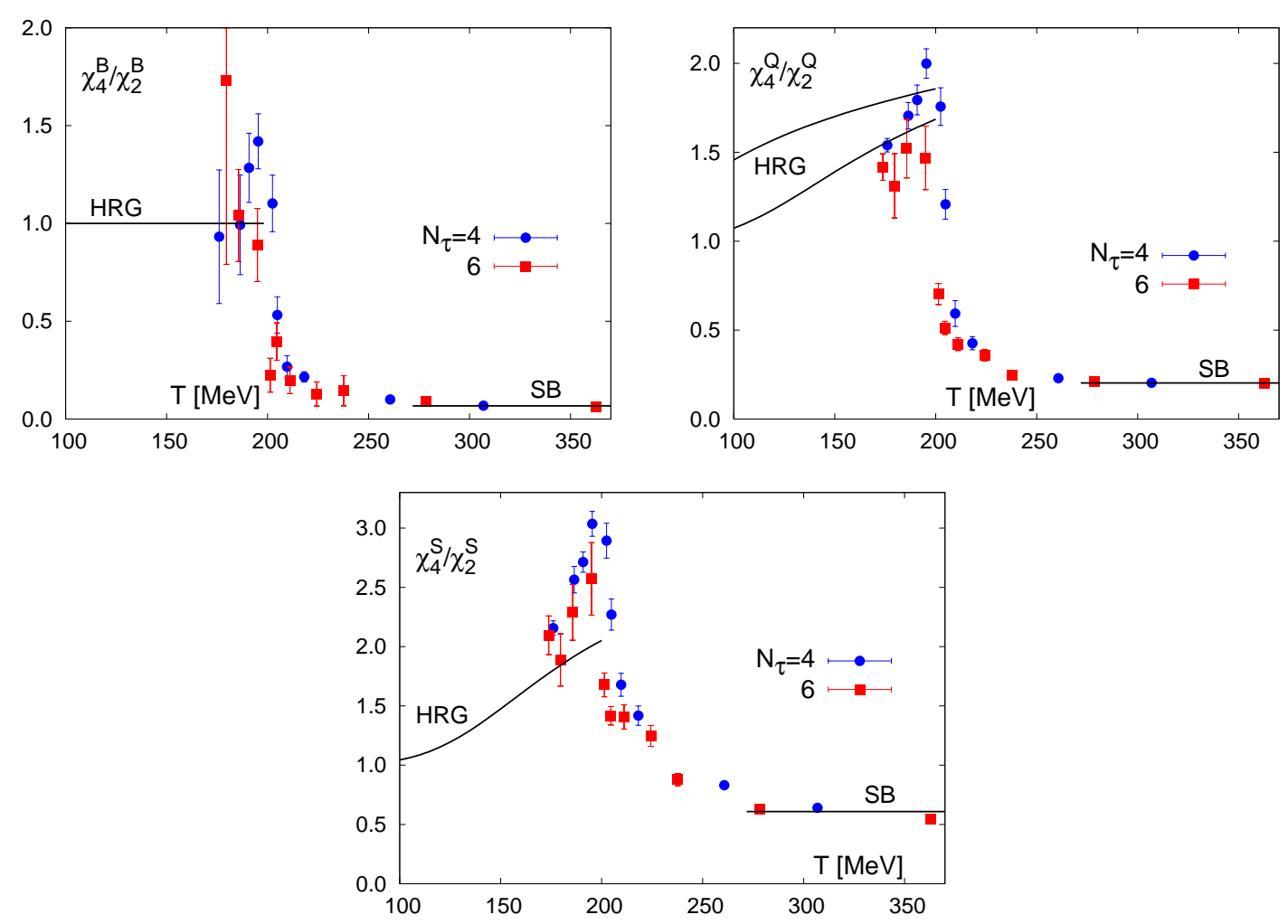

Figure 5: Ratio of quartic and quadratic fluctuations of baryon number $(B)$, strangeness $(S)$ and electric charge $(Q)$. The two curves of the HRG model in the top right figure correspond to charge fluctuations with physical pions (upper) and infinitely heavy pions (lower curve).

In practice, we can not perform the limit $n \rightarrow \infty$, instead we consider the first few approximations $\rho_{n}(T)$, with $n \lesssim 6$. The second approximation for $\rho(T)$ is given by $\rho_{2}(T) \equiv \sqrt{c_{2}^{B}(T) / c_{4}^{B}(T)}=$ $\sqrt{12} / \sqrt{\chi_{4}^{B} / \chi_{2}^{B}}$. We see that $\chi_{4}^{B} / \chi_{2}^{B}$ as shown in Fig. 0 is closely related to the radius of convergence. If this quantity develops a peak, which increases above the resonance gas value of $\chi_{4}^{B} / \chi_{2}^{B}=1$, one might expect the critical point to be found at $\left(\mu_{B} / T\right)^{\text {crit }} \lesssim \sqrt{12}$. Of course, higher order approximations for the radius of convergence are needed to substantiate such an estimate and to establish the existence of a critical point.

Our results are at present not conclusive, as the $N_{\tau}=6$ data shown in Fig. 5 (left) suffers from insufficient statistics and temperature resolution. Note, however, that the peak in the baryon number fluctuation ratio $\chi_{4}^{B} / \chi_{2}^{B}$ will develop in the chiral limit as can be seen from a scaling analysis of the free energy and has recently also shown in a chiral model calculation [16].

\section{Conclusions}

We have calculated corrections to the equation of state arising from a non-zero baryon chemical potential, by means of a Taylor expansion of the pressure. Within this framework we calculated the isentropic equation of state along lines of constant entropy per baryon number $\left(s / n_{B}\right)$ for RHIC, 

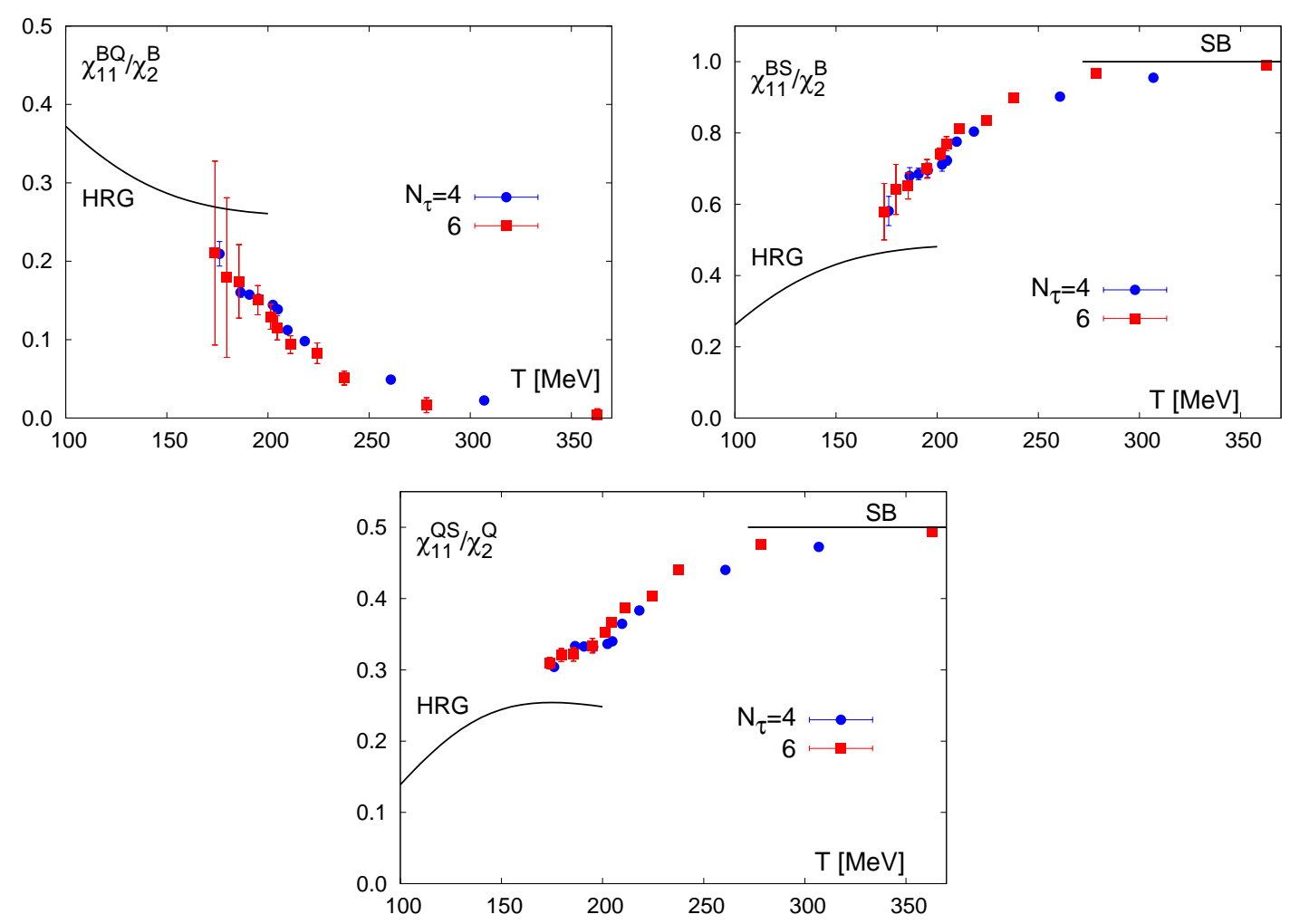

Figure 6: Pairwise correlatios of the conserved charges baryon number (B), electric charge (Q) and strangeness (S) as function of the temperature, normalized to the quadratic fluctuations of B and Q respectively.

SPS and AGS (FAIR) energies. Within our current, preliminary, analysis we find the softest point of the equation of state to be independent of $s / n_{B}$.

Furthermore, we have analyzed the quadratic and quartic fluctuations of baryon number, electric charge and strangeness, as well their ratios. We find these quantities to be in good agreement with the free gas results at temperatures of $T>1.5 T_{c}$. Below $T_{c}$, qualitative features of the resonance gas are reproduced. This ratio for the baryon number is closely related to the second approximation of the convergence radius of the Taylor series of the pressure with respect to the baryon chemical potential.

\section{Acknowledgments}

We thank all members of the RBC-Bielefeld Collaboration for helpful discussions and comments. This work has been supported in part by contracts DE-AC02-98CH10886 and DE-FG0292ER40699 with the U.S. Department of Energy. Numerical simulations have been performed on the QCDOC super-computer of the RIKEN-BNL research center, the DOE funded QCDOC at Brookhaven National Laboratory (BNL) and the apeNEXT installation at the University of Bielefeld. 


\section{References}

[1] M.P. Lombardo, J. Phys. G 35 (2008) 104019;

C. Schmidt, PoS LAT2006 (2006) 021.

[2] C. R. Allton, M. Doring, S. Ejiri, S.J. Hands, O. Kaczmarek, F. Karsch, E. Laermann, K. Redlich, Phys. Rev D 71 (2005) 054508.

[3] C. R. Allton et al., Phys. Rev. D 66 (2002) 074507.

[4] C. Miao and C. Schmidt, PoS LAT2007 (2007) 175.

[5] M. Cheng et al., Phys. Rev. D 77 (2008) 014511.

[6] U. M. Heller, F. Karsch and B. Sturm, Phys. Rev. D 60 (1999) 114502.

[7] P. Hegde, F. Karsch, E. Laermann and S. Shcheredin, Eur. Phys. J. C 55 (2008) 423.

[8] C. Bernard et al., Phys. Rev. D 77 (2008) 014503.

[9] R. V. Gavai and S. Gupta, Phys. Rev. D 71 (2005) 114014; R. V. Gavai and S. Gupta, arXiv:0806.2233 [hep-lat]; S. Gupta this proceedings.

[10] S. Ejiri, F. Karsch, E. Laermann and C. Schmidt, Phys. Rev. D 73 (2006) 054506.

[11] J. Cleymans and K. Redlich, Phys. Rev. Lett. 81 (1998) 5284;

J. Cleymans and K. Redlich, Phys. Rev. C 60 (1999) 054908.

[12] J. Cleymans, H. Oeschler and K. Redlich, S. Wheaton, Phys. Rev. C 73 (2006) 034905.

[13] C. M. Hung and E. Shuryak, Phys. Rev. D 57 (1998) 1891.

[14] V. D. Toneev, J. Cleymans, E. G. Nikonov, K. Redlich and A. A. Shanenko, J. Phys. G 27 (2001) 827.

[15] M. Cheng et al., Phys. Rev. D 74 (2006) 054507.

[16] B. Stokic, B. Friman and K. Redlich, arXiv:0809.3129 [hep-ph]. 